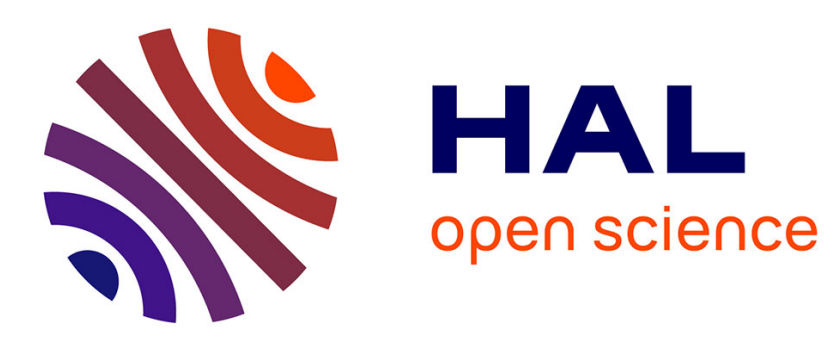

\title{
Entropy production and non-linear hydrodynamic equations of superfluid $3 \mathrm{He}-\mathrm{A}$
}

D. Lhuillier

\section{To cite this version:}

D. Lhuillier. Entropy production and non-linear hydrodynamic equations of superfluid 3He-A. Journal de Physique Lettres, 1977, 38 (5), pp.121-126. 10.1051/jphyslet:01977003805012100 . jpa-00231341

\section{HAL Id: jpa-00231341 https://hal.science/jpa-00231341}

Submitted on 1 Jan 1977

HAL is a multi-disciplinary open access archive for the deposit and dissemination of scientific research documents, whether they are published or not. The documents may come from teaching and research institutions in France or abroad, or from public or private research centers.
L'archive ouverte pluridisciplinaire HAL, est destinée au dépôt et à la diffusion de documents scientifiques de niveau recherche, publiés ou non, émanant des établissements d'enseignement et de recherche français ou étrangers, des laboratoires publics ou privés. 
Classification

Physics Abstracts

$1.680-6.305-7.730$

\title{
ENTROPY PRODUCTION AND NON-LINEAR HYDRODYNAMIC EQUATIONS OF SUPERFLUID ${ }^{3} \mathrm{He}-\mathrm{A}$
}

\author{
D. LHUILLIER \\ Laboratoire d'Aérodynamique, Bâtiment 502, 91405 Orsay Cedex, France
}

(Reçu le 25 novembre 1976, accepté le 28 janvier 1977)

\begin{abstract}
Résumé. - Utilisant une méthode semblable à celle de Khalatnikov pour le ${ }^{4} \mathrm{He}$, nous calculons l'expression de la production d'entropie du ${ }^{3} \mathrm{He}-\mathrm{A}$ superfluide et nous en déduisons les équations hydrodynamiques non linéaires de ce système. Nous comparons ces résultats à ceux des théories linéaires existantes.
\end{abstract}

\begin{abstract}
In a way similar to that used by Khalatnikov in ${ }^{4} \mathrm{He}$, we determine the expression of the entropy production of superfluid ${ }^{3} \mathrm{He}-\mathrm{A}$ and deduce the non-linear hydrodynamic equations of this peculiar fluid. We compare our results with those of previous linear theories.
\end{abstract}

The expression of the entropy production (or dissipation function) in the starting point for the determination of the hydrodynamic equations of any system. This entropy production is easily obtained in the approximation where we neglect terms which are non-linear in the velocities [1]. If we want to take them into account some kind of systematic method is required, the prototype of which has been devised by Khalatnikov [2] for superfluid ${ }^{4} \mathrm{He}$. Here we use a slightly modified form of this technique to determine the entropy production of the so-called A phase of superfluid ${ }^{3} \mathrm{He}$ and to deduce the non-linear nondissipative as well as dissipative hydrodynamic equations.

1. The Gibbs relation of superfluids. - A simple fluid is described by five thermodynamic variables as for instance its mass density $\rho$, entropy density $S$ and the three components of the momentum density $g$. Its energy may be written as

$$
\varepsilon=\varepsilon_{0}(\rho, S)+\frac{g^{2}}{2 \rho}
$$

where $\varepsilon_{0}$ is the internal energy. For more ordered fluids additional thermodynamic variables must be introduced which are related to the order parameter $X$ that describes the broken symmetry [1]; let us call them $x^{\alpha}(X)$. The originality of superfluids is that some of these $x^{\alpha}(X)$ behave like velocities. More precisely, from a superfluid order parameter you can build up a velocity $\mathrm{V}_{\mathrm{s}}(X)$ satisfying the usual transformation law

$$
\mathbf{V}_{\mathrm{s} 1}=\mathbf{V}_{\mathrm{s} 2}+\mathbf{u}
$$

between two galilean frames labelled 1 and 2 and moving with a relative velocity $\mathbf{u}$. Some care is needed when introducing $\mathbf{V}_{\mathrm{s}}(X)$ and $x^{\alpha}(X)$ into the internal energy. The only way to satisfy to the requirement of galilean invariance is to write

$$
\varepsilon=\varepsilon_{0}\left(\rho, S, x^{\alpha}(X), \mathbf{g}-\rho \mathbf{V}_{\mathrm{s}}(X)\right)+\frac{g^{2}}{2 \rho}
$$

where the various $x^{\alpha}(X)$ are supposed to be galilean invariants. If we now define the conjugate parameters $\mu, T, F^{\alpha}$ and $\gamma$ by

$\mathrm{d} \varepsilon_{0}=\mu \mathrm{d} \rho+T \mathrm{~d} S+\sum_{\alpha} F^{\alpha} \mathrm{d} x^{\alpha}(X)+\gamma \cdot \mathrm{d}\left(\mathbf{g}-\rho \mathbf{V}_{\mathrm{s}}(X)\right)$

we see that eqs. (2) and (3) lead to the following form of the Gibbs relation for superfluids

$$
\begin{aligned}
\mathrm{d} \varepsilon= & \left(\mu-\frac{V^{2}}{2}-\gamma \cdot \mathbf{V}_{\mathrm{s}}(X)\right) \mathrm{d} \rho+T \mathrm{~d} S+ \\
& +(\mathbf{V}+\gamma) \cdot \mathrm{dg}+\sum_{\alpha} F^{\alpha} \mathrm{d} x^{\alpha}(X)-\rho \gamma \cdot \mathrm{d} \mathbf{V}_{\mathrm{s}}(X)
\end{aligned}
$$

where we have introduced the velocity $\mathbf{V}=\mathbf{g} / \rho$. We may thus say that a superfluid is distinguished from other ordered fluids by the presence of the intrinsic velocity $\gamma$. Endeed when $\gamma$ is zero, we recover the usual Gibbs relation for non-superfluid ordered fluids (i.e. eq. (2.3) of ref. [1]). We shall see that the sum $\mathbf{V}+\gamma$ is in fact what is called $\mathbf{V}_{\mathbf{n}}$ in the Landau terminology but for reasons that will soon become clear we keep on using $\gamma$. Let us also notice that the variables $x^{\alpha}$ 
and $\mathbf{V}_{\mathrm{s}}$ appearing in eq. (4) are all a function of the order parameter $X$; we must keep this in mind since this means that the variations $d x^{\alpha}$ and $d V_{s}$ are not independent and that the unique variable is $X$. Eventually we can have an ordered system with $\mathbf{V}_{\mathrm{s}}$ deriving from a first order parameter $X_{1}$ and the variables $x^{\alpha}$ from a second one $X_{2}$. But one single order parameter is sufficient for superfluid ${ }^{3} \mathrm{He}-\mathrm{A}$ and in this case eq. (4) provides us with the relevant Gibbs relation. The ${ }^{3} \mathrm{He}-\mathrm{A}$ order parameter (at least its spatial part) is a complex vector $\Delta$ [4], transforming in a change of galilean frame like

$\Delta_{1}=\Delta_{2} \mathrm{e}^{i \theta} \quad$ with $\quad \theta=\frac{2 m_{3}}{\hbar}\left(\mathbf{u} \cdot \mathbf{r}_{2}+\frac{1}{2} u^{2} t_{2}\right)$.

From this transformation law $\left({ }^{1}\right)$ it appears that we can define the superfluid velocity as

$$
\mathbf{V}_{\mathrm{s}}(\boldsymbol{\Delta})=\frac{\hbar}{4 m_{3} i|\Delta|^{2}}\left(\Delta_{j}^{*} \nabla \Delta_{j}-\Delta_{j} \nabla \Delta_{j}^{*}\right) .
$$

However, with the complex vector $\Delta$ you can also obtain galilean invariant velocities, and the sum of expression (6) with any combination of these intrinsic velocities is also a valuable candidate for $\mathbf{V}_{\mathbf{s}}(\Delta)$. In this Letter we choose to consider the intrinsic velocities as pertaining to the $x^{\alpha}(\boldsymbol{\Delta})$, hence we adopt for $\mathbf{V}_{\mathrm{s}}(\boldsymbol{\Delta})$ the simplest choice given by eq. (6). We shall see that the final expression of the entropy production is - happily enough - independent of this particular choice. Let us now discuss on the various possible $x^{\alpha}(\Delta)$. If we remember that they must be galilean invariants we deduce from eq. (5) that they must involve an equal number of $\Delta$ and $\Delta^{*}$ and the simplest ones are $\Delta . \Delta^{*}, \Delta \times \Delta^{*}, \Delta^{2} \Delta^{* 2},\left(\Delta . \Delta^{*}\right) \Delta \times \Delta^{*}$, $\left(\Delta \times \Delta^{*}\right)^{2}$, etc., and their derivatives $\nabla\left(\Delta . \Delta^{*}\right)$, $\nabla .\left(\Delta \times \Delta^{*}\right), \nabla \times\left(\Delta \times \Delta^{*}\right)$, etc. But the structure of $\Delta$ is such that $\Delta^{2}=\Delta^{* 2}=0$ and $\Delta . \Delta^{*}=$ constant [5]. For this reason these terms (as well as $\left(\Delta \times \Delta^{*}\right)^{2}$ which is also constant) must be eliminated from the list and we are left with $\Delta \times \Delta^{*}$ and its derivatives as the only possible $x^{\alpha}(\Delta)$. If we introduce the real vector 1 defined as [4]

$$
1=\frac{i}{2} \Delta \times \Delta^{*}
$$

the general Gibbs relation eq. (4) becomes for superfluid ${ }^{3} \mathrm{He}-\mathrm{A}$

$$
\begin{aligned}
\mathrm{d} \varepsilon=\left(\mu-\frac{V^{2}}{2}-\right. & \left.\gamma \cdot \mathbf{V}_{\mathrm{s}}(\Delta)\right) \mathrm{d} \rho+T \mathrm{~d} S+ \\
& +(\mathbf{V}+\gamma) \cdot \mathrm{d} \mathbf{g}-\rho \gamma \cdot \mathrm{d} \mathbf{V}_{\mathrm{s}}(\boldsymbol{\Delta}) \\
& +\phi_{i j} \mathrm{~d}\left(\frac{\partial \rho_{i}(\Delta)}{\partial x_{j}}\right)+\lambda_{i} \mathrm{~d} l_{i}(\Delta) .
\end{aligned}
$$

( $\left.{ }^{1}\right)$ This is a consequence of the gauge-invariant property of the microscopic equation acting on $\Delta$.
Alternatively, using the definitions (6) and (7) of $\mathbf{V}_{\mathbf{s}}$ and $\mathbf{l}$ and putting $|\boldsymbol{\Delta}|^{2}=2$, we get the more fundamental expression involving $\Delta$ only

$$
\begin{aligned}
& \mathrm{d} \varepsilon=\left(\mu-\frac{V^{2}}{2}-\gamma \cdot \mathbf{V}_{s}(\Delta)\right) \mathrm{d} \rho+T \mathrm{~d} S+ \\
& +(\mathbf{V}+\gamma) \cdot \mathrm{dg}+\left(h_{i j}^{*} \mathrm{~d}\left(\frac{\partial \Delta_{i}}{\partial x_{j}}\right)+f_{i}^{*} \mathrm{~d} \Delta_{i}+\text { c.c. }\right)
\end{aligned}
$$

with

$f_{i}=\frac{i}{2}\left[\varepsilon_{i p q}\left(\lambda_{p} \Delta_{q}+\phi_{p r} \frac{\partial \Delta_{q}}{\partial x_{r}}\right)+\frac{\hbar}{4 m_{3}} \rho \gamma_{p} \frac{\partial \Delta_{i}}{\partial x_{p}}\right]$

and

$$
h_{i j}=\frac{i}{2}\left[\varepsilon_{i p q} \phi_{p j} \Delta_{q}-\frac{\hbar}{4 m_{3}} \rho \gamma_{j} \Delta_{i}\right] .
$$

Let us stress that the terms $\phi_{i j}$ and $\lambda_{i}$ appearing in eqs. (8), (10) and (11) are not a priori supposed to be analogous to the Frank terms in nematics. We shall even see that they are likely to be different.

2. The thermodynamic equilibrium conditions. Let us consider a bath of superfluid ${ }^{3} \mathrm{He}-\mathrm{A}$ to which no heat or work is supplied. At equilibrium its entropy will be a maximum for given values of its energy, mass, momentum and moment of momentum. In the case of ${ }^{3} \mathrm{He}-\mathrm{A}$ the entropy also depends on the order parameter $\Delta$ verifying $\Delta^{2}=\Delta^{* 2}=0$ and $\Delta \cdot \Delta^{*}=$ constant. Then, the condition of maximum of entropy may be expressed as [6]

$$
\begin{aligned}
\delta \int[S & +C_{a} \rho+C_{b} \varepsilon+\mathbf{C}_{c} \cdot \mathbf{g}+\mathbf{C}_{d} \cdot(\mathbf{r} \times \mathbf{g}) \\
& \left.+C_{1}\left(\Delta^{2}+\Delta^{* 2}\right)+C_{2} \Delta \cdot \Delta^{*}\right] \mathrm{d} V=0
\end{aligned}
$$

where the $C_{i}$ are (real) Lagrange multipliers. Using the Gibbs relation (9) to express $\delta S$ as a function of the independent variations $\delta \rho, \delta \varepsilon, \delta \mathbf{g}$ and $\delta \Delta$, and cancelling the coefficients of these variations we find the following equilibrium conditions

$$
\begin{aligned}
\mu-\frac{V^{2}}{2}-\gamma \cdot \mathbf{V}_{\mathbf{s}} & =\mu_{0} \\
T & =T_{0} \\
\mathbf{V}+\gamma & =\mathbf{u}+\mathbf{\Omega} \times \mathbf{r}
\end{aligned}
$$

and

$$
-f_{i}+\frac{\partial h_{i j}}{\partial x_{j}}=-C_{1} \Delta_{i}^{*}-C_{2} \Delta_{i}
$$

In eqs. (13) to (15), the terms $\mu_{0}, T_{0}, \mathbf{u}$ and $\Omega$ are constant and the last equation may be written, with the help of eqs. (10) and (11), as

$$
\begin{aligned}
\Delta \times \mathbf{h}+\frac{\hbar \rho}{2 m_{3}}(\boldsymbol{\gamma} \cdot \boldsymbol{\nabla}) \boldsymbol{\Delta}+\frac{\hbar}{4 m_{3}} & (\boldsymbol{\nabla} \cdot \rho \boldsymbol{\gamma}) \boldsymbol{\Delta}= \\
& =i \lambda \Delta+i \lambda^{\prime} \Delta^{*}
\end{aligned}
$$


where $\lambda$ and $\lambda^{\prime}$ are real numbers and where $\mathbf{h}$ is defined by analogy with nematics as

$$
h_{i}=+\frac{\partial \phi_{i j}}{\partial x_{j}}-\lambda_{i}
$$

The conditions $\Delta^{2}=0$ and $\Delta . \Delta^{*}=$ constant imply that $\lambda^{\prime}=0$. Moreover it may be shown by projecting eq. (16) on three orthogonal axes that this equilibrium condition splits into two independent parts

$$
\nabla \cdot \rho \gamma=0
$$

and

$$
\Delta \times \mathbf{h}+\frac{\hbar \rho}{2 m_{3}}(\boldsymbol{\gamma} \cdot \boldsymbol{\nabla}) \Delta=i \lambda \Delta
$$

To achieve the interpretation of the last equation we split it into its real and imaginary parts which are then solved for $\mathbf{h}$ and lead to

$$
\mathbf{h}+\frac{\rho \hbar}{2 m_{3}}(\gamma \cdot \nabla) \mathbf{l} \times \mathbf{l}=\left(\lambda-\rho \gamma \cdot \mathbf{V}_{\mathbf{s}}\right) \mathbf{l}
$$

This result explicitly shows the connexion between $\gamma$ and $\mathbf{h}$, which is itself a manifestation of the dependence of both $V_{s}$ and $\mathbf{l}$ on $\Delta$. When $\gamma=0$ eq. (20) merges into the equilibrium condition of nematics [3, 7]. When $\mathbf{h}=\mathbf{0}$ (i.e. $\mathbf{l}=$ constant) we are left with the condition $\nabla . \rho \gamma=0$ of superfluid ${ }^{4} \mathrm{He}$ [6]. But it is evident that in every other situation there is an interplay between $\boldsymbol{\gamma}$ and $\mathbf{h}$, and superfluid ${ }^{3} \mathrm{He}-\mathrm{A}$ in no way behaves as a superposition of a nematic and a superfluid but rather as an intimate combination of both.

From the equilibrium conditions eqs. (13), (14), (15), (18) and (20) we can foresee that the thermodynamic forces $X_{i}$ [8] that will appear in the entropy production will be of the form

$$
\begin{gathered}
\nabla T, \quad \nabla\left(\mu-\frac{V^{2}}{2}-\gamma \cdot \mathbf{V}_{\mathbf{s}}(\Delta)\right), \quad \nabla \cdot \rho \gamma, \\
\frac{\partial\left(V_{i}+\gamma_{i}\right)}{\partial x_{j}}+\frac{\partial\left(V_{j}+\gamma_{j}\right)}{\partial x_{i}} \text { and } \mathbf{h}+\frac{\rho \hbar}{2 m_{3}}(\gamma \cdot \nabla) \mathbf{l} \times \mathbf{l} .
\end{gathered}
$$

In particular there cannot exist any dissipation involving $\nabla \times(\mathbf{V}+\gamma)=\nabla \times \mathbf{V}_{n}$.

\section{The equations of motion of the state variables. -} As already stressed, a thermodynamic state of ${ }^{3} \mathrm{He}-\mathrm{A}$ is completely specified once we know the values of the state variables $\rho, \varepsilon, g$ and $\Delta$. If we want to describe the time variation of the state we must know the equations of motion of our set of variables. As to $\rho$, $\varepsilon$ and $\mathbf{g}$ they satisfy to conservation laws (in the absence of external forces) which must be written so as to display galilean invariance

$$
\begin{aligned}
& \frac{\partial \rho}{\partial t}+\nabla \cdot \rho \mathbf{V}=0 \\
& \frac{\partial \mathbf{g}}{\partial t}+\nabla \cdot\left(\overrightarrow{\overrightarrow{\boldsymbol{\sigma}}_{0}}+p \overrightarrow{\overrightarrow{\mathbf{V}}} \overrightarrow{\overrightarrow{\mathbf{V}}}\right)=0 \\
& \frac{\partial \varepsilon}{\partial t}+\nabla \cdot\left(\mathbf{Q}_{0}+\mathbf{V} \cdot \overrightarrow{\overrightarrow{\boldsymbol{\sigma}}}_{0}+\varepsilon \mathbf{V}\right)=0
\end{aligned}
$$

$\overrightarrow{\boldsymbol{\sigma}}_{0}$ and $\mathbf{Q}_{0}$ are respectively the (unknown) momentum flux and energy flux in the frame where $\mathbf{V}=\mathbf{g} / \rho=0$. We assume that conservation of moment of momentum is automatically satisfied. This is usually done by assuming that $\overrightarrow{\mathrm{\sigma}}_{0}$ is symmetrical ; it appears however that in the case of nematics (and ${ }^{3} \mathrm{He}-\mathrm{A}$ ) a more fruitful assumption is to suppose that the antisymmetric part of $\overrightarrow{\boldsymbol{\sigma}}_{0}$ can be written in the form of a divergence (of a third order tensor). This hypothesis is implicitly contained in the work of de Gennes [3] and we use it mainly to avoid the introduction of a hypothetic intrinsic angular momentum.

Our chief task is now to derive an equation for $\boldsymbol{\Delta}$. In a change of galilean frames $\Delta$ behaves like in eq. (6) and we deduce that

$$
\begin{aligned}
& \Delta_{1 k}^{*}\left[\frac{\partial \Delta_{1 j}}{\partial t_{1}}+V_{1 p} \nabla_{1 p} \Delta_{1 j}-\frac{2 m_{j i}}{\hbar} \frac{V_{1}^{2}}{2} \Delta_{1 j}\right]= \\
& =\Delta_{2 k}^{*}\left[\frac{\partial \Delta_{2 j}}{\partial t_{2}}+V_{2 p} \nabla_{2 p} \Delta_{2 j}-\frac{2 m_{3 i}}{\hbar} \frac{V_{2}^{2}}{2} \Delta_{2 j}\right]
\end{aligned}
$$

for any velocity satisfying to eq. (1). We then conclude that the equation of motion of $\Delta$ must be of the general form

$$
\Delta_{k}^{*}\left[\frac{\partial \Delta_{j}}{\partial t}+(\mathbf{V} . \nabla) \Delta_{j}-\frac{2 i m_{3}}{\hbar} \frac{V^{2}}{2} \Delta_{j}\right]=N_{0 k j}
$$

where $\overrightarrow{\mathbf{N}}_{0}$ is a galilean-invariant tensor. Moreover the $\Delta$ equation must be compatible with $\Delta . \Delta^{*}=$ constant and this is satisfied only if the trace $N_{0 j j}$ is null or is a pure imaginary number. Lastly we require the equation of evolution of $\Delta \times \Delta^{*}$ not only be galileaninvariant but also invariant when we pass from a galilean frame to a rotating frame. We infer the latter condition because $\Delta \times \Delta^{*}$ is invariant both in translation and rotation $\left({ }^{1}\right)$ and we think that its equation of motion must be invariant in the corresponding operations. It may be shown that our requirement is satisfied for

$$
N_{0 k j}=\Delta_{k}^{*}\left[\Delta \times\left(\Omega_{0}-\frac{1}{2} \nabla \times \mathbf{V}\right)\right]_{j}
$$

We remark that this particular $\overrightarrow{\mathbf{N}}_{0}$ has zero trace and is thus compatible with $\boldsymbol{\Delta} . \Delta^{*}=$ constant. Postu- 


\section{TABLE I}

Structure of the entropy production of superfluid ${ }^{3} \mathrm{He}-\mathrm{A}$

$$
\sigma=\frac{1}{T} \sum_{i} J_{i} X_{i}
$$

nature of dissipation

thermal

shear flow of $\mathbf{V}+\gamma \equiv \mathbf{V}_{n}$

rotation of $\Delta$ around l

$$
\begin{gathered}
-J_{i} \\
- \\
\mathbf{S}_{0}-S \gamma
\end{gathered}
$$

$$
\left(\sigma_{0 i j}\right)^{\mathrm{sym}}-p \delta_{i j}-\left(\sigma_{i j}^{d}\right)^{\mathrm{sym}}
$$

$$
\mu+\frac{\hbar}{2 m_{3}}\left(\mathbf{\Omega}_{0}+\frac{1}{2} \nabla \times \gamma\right) \cdot \mathbf{l}
$$

$$
\mathbf{\Omega}_{0} \times \mathbf{l}-(\gamma \cdot \nabla) \mathbf{l}-\frac{1}{2} \mathbf{l} \times(\nabla \times \gamma)
$$

$$
\left(\sigma_{0 i j}\right)^{a}-\left(\sigma_{i j}^{d}\right)^{a}-\left(h_{i} l_{j}\right)^{a}
$$

$X_{i}$

$\nabla T$ rotation of $\Delta$ perpendicular to I

not allowed lating that no other terms appear in $\overrightarrow{\vec{N}}_{0}\left({ }^{2}\right)$, we intro-
duce it in eq. (25). Eliminating the factor $\Delta_{k}^{*}$ which is now unimportant, we obtain the desired equation of evolution

$$
\begin{aligned}
\frac{\partial \Delta}{\partial t}+(\mathbf{V} . \boldsymbol{\nabla}) \boldsymbol{\Delta}+\Delta \times \frac{1}{2}(\boldsymbol{\nabla} \times \mathbf{V}) & -\frac{2 i m_{3}}{\hbar} \frac{V^{2}}{2} \Delta= \\
& =\Delta \times \mathbf{\Omega}_{0} .
\end{aligned}
$$

An analogous form of equation has been found by Delrieu [5] with arguments on gauge invariance (cf. footnote 1). In this equation the unknown is $\boldsymbol{\Omega}_{0}$ and the only requirement on $\mathbf{V}$ is to satisfy eq. (1). For definiteness we adopt for $\mathbf{V}$ the mass flux velocity $\mathrm{g} / \rho$, that is to say the velocity already appearing in eqs. (22) to (24).

4. The entropy production. - In eqs. (23), and (26) the terms $\overrightarrow{\boldsymbol{\sigma}}_{0}, \mathbf{Q}_{0}$ and $\boldsymbol{\Omega}_{0}$ are unknown. To determine them we use a slightly modified form of a method proposed by Khalatnikov [2]. From the Gibbs relation eq. (9) we write

$$
\begin{aligned}
\frac{\partial S}{\partial t}= & \frac{1}{T} \frac{\partial \varepsilon}{\partial t}-\frac{\mu-\frac{V^{2}}{2}-\gamma \cdot \mathbf{V}_{\mathrm{s}}}{T} \frac{\partial \rho}{\partial t}-\frac{\mathbf{v}+\gamma}{T} \cdot \frac{\partial \mathbf{g}}{\partial t}- \\
& -\frac{1}{T}\left[h_{i j}^{*} \frac{\partial}{\partial x_{j}}\left(\frac{\partial \Delta_{i}}{\partial t}\right)+f_{i}^{*} \frac{\partial \Delta_{i}}{\partial t}+\text { c.c. }\right] .
\end{aligned}
$$

$\left({ }^{2}\right)$ This corresponds to the statement by Hall [9] that the motion of the order parameter can be entirely described by an instantaneous rotation vector, and $\boldsymbol{\Omega}_{0}-\frac{1}{2} \boldsymbol{\nabla} \times \mathbf{V}$ is in fact analogous to the $\mathbf{C}$ vector of Hall.
We then replace time derivatives by spatial derivatives with the help of eqs. (22), (23), (24) and (26), and finally try to put the result in the form

$$
\frac{\partial S}{\partial t}+\nabla \cdot\left(\mathbf{S}_{0}+S \mathbf{V}\right)=\frac{1}{T} \sum_{i} J_{i} X_{i}
$$

where the $X_{i}$ are the thermodynamic forces we determined in $\S 2$ (eqs. (21)). At the end of the calculation we find expressions for the entropy flux $S_{0}$ and the $J_{i}$ as a function of $\overrightarrow{\boldsymbol{\sigma}}_{0}, \mathbf{Q}_{0}$ and $\mathbf{\Omega}_{0}$. Then, from the positivity of the entropy production together with the standard methods of thermodynamics of irreversible processes [8] we deduce the expressions of the unknown terms first for non-dissipative situations and then for dissipative ones. We shall avoid the detail of the long calculation which leads from eq. (27) to eq. (28) and simply quote the final result.

The entropy flux is found to be

$$
\begin{aligned}
T \mathbf{S}_{0}= & \mathbf{Q}_{0}-\gamma \cdot\left(\overrightarrow{\overrightarrow{\boldsymbol{\sigma}}_{0}}-\overrightarrow{\overrightarrow{\mathbf{I}}}\right)-\rho \gamma \times \\
& \times\left[\mu+\frac{\hbar}{2 m_{3}}\left(\mathbf{\Omega}_{0}+\frac{1}{2} \boldsymbol{\nabla} \times \gamma\right) \cdot \mathbf{l}\right] \\
& +\left[\mathbf{I} \times\left(\mathbf{\Omega}_{0}-\frac{1}{2} \nabla \times \mathbf{V}\right)+(\gamma \cdot \Delta) \mathbf{I}\right] \cdot \overrightarrow{\overrightarrow{\boldsymbol{\phi}}}
\end{aligned}
$$

where $\overrightarrow{\vec{I}}$ represents the unit tensor and the pressure $p$ is defined as

$$
p=\rho \mu+T S+\gamma \cdot\left(\mathbf{g}-\rho \mathbf{V}_{\mathrm{s}}\right)-\varepsilon_{0} .
$$

The fluxes $J_{i}$ corresponding to the forces $X_{i}$ in the entropy production are presented in table I (note the minus sign of $J_{i}$ ) : 
In the above table, $\overrightarrow{\vec{\sigma}^{d}}$ represents a generalized distortion stress tensor $[3,7]$ defined as

$$
\sigma_{i j}^{d}=\phi_{k j} \frac{\partial \rho_{k}}{\partial x_{i}}+\left(g_{i}-\rho V_{\mathrm{si}}\right) \gamma_{j}
$$

There is one term which should not have appeared among the $X_{i}$ of table I, namely the last one. This is because $\boldsymbol{\nabla} \times(\mathbf{V}+\boldsymbol{\gamma})$ may be non-zero at equilibrium and that the equilibrium entropy production must be null. The only way to solve this problem is to give to the antisymmetric part of $\overrightarrow{\vec{\sigma}}_{0}$ the well-defined value (valuable in non-dissipative as well as in dissipative situations)

$$
\left(\sigma_{0 i j}\right)^{a}=\left(\sigma_{i j}^{d}+h_{i} \rho_{j}\right)^{a}
$$

equivalent to eq. (5.42) of de Gennes [3]. Moreover for the angular momentum $\mathbf{r} \times \mathbf{g}$ to be conserved despite the presence of $\overrightarrow{\boldsymbol{\sigma}}_{0}^{a}$, this antisymmetric part must be the divergence of a third-order tensor. Such a requirement is satisfied if the condition

$$
\left(\sigma_{i j}^{d}\right)^{a}=\left(\lambda_{i} \rho_{j}+\phi_{i k} \frac{\partial \rho_{j}}{\partial x_{k}}\right)^{a}
$$

is obeyed by the thermodynamic derivatives $\lambda_{i}, \phi_{i j}$ and $\gamma_{i}$. And in fact if condition (33) is satisfied one can write

$$
\left(\sigma_{0 i j}\right)^{a}=\frac{\partial}{\partial x_{k}}\left(\phi_{i k} \rho_{j}\right)^{a}
$$

In a nematic liquid crystal $\boldsymbol{\gamma}$ is null and condition (33) (which expresses the invariance of the Frank energy in the rotation of both the position and the director $[3,7])$ is satisfied by the Frank terms $\lambda_{i}^{\mathbf{F}}$ and $\phi_{i j}^{\mathbf{F}}$. We guess that eq. (33) is a natural generalization to the case where $\gamma$ is non-zero, but it must be stressed that such a condition cannot be satisfied by $\lambda_{i}^{F}$ and $\phi_{i j}^{F}$ except if $\mathbf{g}-\rho \mathbf{V}_{\mathbf{s}}$ and $\boldsymbol{\gamma}$ always stay parallel, as in isotropic ${ }^{4} \mathrm{He}$. Lastly we notice that table I contains the special cases of ${ }^{4} \mathrm{He}$ and nematics : For $\gamma=0$ we recover the entropy production of a nematic. At variance if we suppose that $\lambda_{i}$ and $\phi_{i j}$ are null (I constant everywhere) we are left with the entropy production of superfluid ${ }^{4} \mathrm{He}$. This was the reason which led us to use $\boldsymbol{\gamma}$ instead of $\mathbf{V}_{n}=\mathbf{V}+\boldsymbol{\gamma}$ all along the calculations.

\section{The non-dissipative hydrodynamic equations. -}

From table I we deduce that the unknown terms $\overrightarrow{\vec{\sigma}}_{0}$, $\mathbf{S}_{0}$ and $\boldsymbol{\Omega}_{\mathbf{0}}$ (together with $\mathbf{Q}_{0}$ related to them by eq. (29)) may be written as

$$
\begin{aligned}
& \text { reactive } \\
& \sigma_{0 i j}=\quad p \delta_{i j}+\sigma_{i j}^{d}+\frac{1}{2}\left(h_{i} \rho_{j}-h_{j} \rho_{i}\right)+ \\
& \mathbf{\Omega}_{0}=\quad-\frac{1}{2} \nabla \times \gamma-(\gamma \cdot \nabla) \mathbf{l} \times \mathbf{l}-\frac{2 m_{3} \mu}{\hbar} \mathbf{1}+ \\
& \mathbf{S}_{0}=\quad S \gamma+ \\
& \sigma_{i j}^{r}+ \\
& \text { dissipative } \\
& \mathbf{\Omega}^{r}+ \\
& \mathbf{\Omega}^{d} \\
& \mathbf{S}^{r}+ \\
& \mathbf{S}^{d} .
\end{aligned}
$$

reactive with

The terms we call reactive with phenomenological coefficients are such that

$$
\sigma_{i j}^{r} A_{i j}+\mathbf{\Omega}^{r} \cdot\left(1 \times \mathbf{h}_{\mathrm{s}}+\frac{\hbar \rho}{2 m_{3}}(\nabla \cdot \rho \gamma)\right)+\mathbf{S}^{r} \cdot \nabla T=0
$$

where we have defined

and

$$
A_{i j}=\frac{1}{2}\left(\frac{\partial V_{i}+\gamma_{i}}{\partial x_{j}}+\frac{\partial V_{j}+\gamma_{j}}{\partial x_{i}}\right)
$$

$$
\mathbf{h}_{\mathbf{s}}=\mathbf{h}+\frac{\hbar \rho}{2 m_{3}}(\gamma \cdot \nabla) \mathbf{l} \times \mathbf{l} .
$$

Moreover they must have the same parity under time-reversal as the pure reactive part and this requirement leads to

$$
\begin{aligned}
& \mathbf{S}^{r}=K \mathbf{l} \times \nabla T \\
& \mathbf{\Omega}^{r}=\beta \mathbf{l} \times\left(\mathbf{l} \times \mathbf{h}_{\mathbf{s}}\right)+2 \alpha \mathbf{l} \times(\overrightarrow{\overrightarrow{\mathbf{A}}} . \mathbf{l}) \\
& \sigma_{i j}^{r}=\varphi_{1}\left[(\mathbf{l} \times \overrightarrow{\overrightarrow{\mathbf{A}}})_{i j}+(i \leftrightarrow j)\right]+\varphi_{2}\left[(\mathbf{l} \times(\overrightarrow{\overrightarrow{\mathbf{A}}} . \mathbf{l}))_{i} l_{j}+(i \leftrightarrow j)\right]+\alpha\left[\left(\mathbf{l} \times\left(\mathbf{l} \times \mathbf{h}_{\mathbf{s}}\right)\right)_{i} l_{j}+(i \leftrightarrow j)\right]
\end{aligned}
$$


where the notation and some of the material have been gathered from Graham [10], Liu [11] and de Gennes [3]. In particular from the above expression of $\boldsymbol{\Omega}^{r}$ we get the non-dissipative equation of motion of the order parameter

$$
\frac{\partial \Delta}{\partial t}+\left(\mathbf{V}_{n} \cdot \nabla\right) \Delta+\Delta \times \omega+\frac{2 i m_{3}}{\hbar}\left(\tilde{\mu}-\mathbf{V}_{n} \cdot \mathbf{V}_{s}\right) \Delta=0
$$

where

and

$$
\begin{aligned}
& \boldsymbol{\omega}=\frac{1}{2} \nabla \times \mathbf{V}_{n}+\beta \mathbf{h}_{\mathrm{s}}+2 \alpha(\overrightarrow{\vec{A}} \cdot \mathbf{l}) \times \mathbf{l} \\
& \tilde{\mu}=\mu+\frac{1}{2} V_{\mathrm{s}}^{2}-\frac{1}{2}\left(\mathbf{V}-\mathbf{V}_{\mathrm{s}}\right)^{2}-\frac{\hbar}{2 m_{3}} \beta \mathbf{h}_{\mathrm{s}} \cdot \mathbf{l},
\end{aligned}
$$

$$
\mathbf{V}_{n}=\mathbf{V}+\gamma
$$

In order to compare our results with those of Graham [10] we need the equations for $V_{s}$ and $\mathbf{l}$. Combining the definitions (6) and (7) with the above equation we obtain

$$
\frac{\partial \mathbf{l}}{\partial t}+\left(\mathbf{V}_{n} \cdot \nabla\right) \mathbf{I}+\mathbf{l} \times \omega=0
$$

and

$\frac{\partial \mathbf{V}_{\mathrm{s}}}{\partial t}-\mathbf{V}_{n} \times\left(\nabla \times \mathbf{V}_{\mathrm{s}}\right)+\nabla \tilde{\mu}+\frac{\hbar l_{j}}{2 m_{3}} \nabla \omega_{j}=0$

Let us now consider states of nearly uniform textures for which $\mathbf{l}(\mathbf{r})=\mathbf{l}_{0}+\delta \mathbf{l}(\mathbf{r})$ and all velocities are infinitesimal. If we neglect the product of two or more of the quantities $\mathbf{V}_{n}, \mathbf{V}_{\mathrm{s}}, \mathbf{V}, \boldsymbol{\gamma}$ and $\delta \mathrm{l}$, we get the linearized equations

$$
\frac{\partial \mathbf{l}}{\partial t}+\mathbf{l}_{0} \times \omega=0
$$

and

$$
\frac{\partial \mathbf{V}_{\mathrm{s}}}{\partial t}+\nabla\left(\mu+\frac{\hbar}{2 m_{3}} \mathbf{l}_{0} \cdot \frac{1}{2} \nabla \times \mathbf{V}_{n}\right)=0
$$

These linearized equations with an irrotational velocity $\mathbf{V}_{\mathrm{s}}$ are exactly those found by Graham [10] and later improved by Liu [11].

6. The dissipative hydrodynamic equations. - We shall sketch only briefly the way one can obtain the dissipative parts of $\overrightarrow{\boldsymbol{\sigma}}_{0}, \mathbf{S}_{0}$ and $\boldsymbol{\Omega}_{\mathbf{0}}$. These dissipative components must be such that

$$
\begin{aligned}
\sigma_{i j}^{d} A_{i j}+ & \left(\mathbf{\Omega}^{d} \times \mathbf{l}\right) \cdot h_{s}+ \\
& +\frac{\hbar}{2 m_{3}}\left(\boldsymbol{\Omega}^{d} \cdot \mathbf{l}\right) \nabla \cdot \rho \gamma+\mathbf{S}^{d} \cdot \nabla T>0 .
\end{aligned}
$$

This expression is similar to that used by Graham and our dissipative terms can be deduced from those he calculated if we replace his $\nabla . \lambda^{\mathrm{s}}$ by $-\nabla . \rho \gamma$ and his $\partial \phi_{i j} / \partial x_{j}$ by $\mathbf{h}_{\mathrm{s}}$. If we limit ourselves to a linear theory (in the above sense) then $\mathbf{h}_{\mathrm{s}}$ becomes $\partial \phi_{i j} / x_{j}$ and the dissipative terms are exactly those of Graham.

I am indebted to J. M. Delrieu for convincing me that $\Delta$ is indeed the best variable on which hydrodynamics of ${ }^{3} \mathrm{He}-\mathrm{A}$ could be based.

Note added in proof : In the expression of the entropy production (Table I) a term has been omitted. Instead of

$$
\left(\sigma_{0 i j}\right)^{a}-\left(\sigma_{i j}^{d}\right)^{a}-\left(h_{i} l_{j}\right)^{a}
$$

one schould read

$$
\left(\sigma_{0 i j}\right)^{a}-\left(\sigma_{i j}^{d}\right)^{a}-\left(h_{i} l_{j}\right)^{a}+\frac{\hbar}{4 m} \varepsilon_{i j p} \frac{\partial}{\partial x_{k}}\left(\rho \gamma_{k} l_{p}\right) .
$$

The expression of the antisymetric part of the momentum flux tensor is then

$$
\left(\sigma_{0 i j}\right)^{a}=\frac{\partial}{\partial x_{k}}\left[\frac{1}{2}\left(\phi_{i k} l_{j}-\phi_{j k} l_{i}\right)-\frac{\hbar}{4 m} \varepsilon_{i j p} \rho \gamma_{k} l_{p}\right] .
$$

The above error was pointed out by Drs C.-R. Hu and T.-L. Ho, and we thank them for sending us their results before publication.

\section{References}

[1] Martin, P. C., Parodi, O. and Pershan, P. S., Phys. Rev. A 6 (1972) 2401.

[2] Khalatnikov, I. M., Introduction to the theory of superfluidity (W. J. Benjamin, New York) 1965.

[3] DE Gennes, P. G., The physics of liquid crystals (Clarendon Press, Oxford) 1974, eq. (3.109).

[4] Anderson, P. W. and Brinkman, W. F., in the Proceedings of the 15th Scottish Summer School, ed. J. Armitage and I. Farquhar (Academic Press, London) 1975.

[5] Delrieu, J. M., J. Physique Lett. 38 (1977) L-127.
[6] See Putterman, S. J., Superfluid hydrodynamics (NorthHolland, Amsterdam) 1974 for the case of superfluid ${ }^{4} \mathrm{He}$.

[7] Forster, D., Lubensky, T. C., Martin, P. C., SWift, J. and Pershan, P., Phys. Rev. Lett. 26 (1971) 1016.

Forster, D., Ann. Phys. 85 (1974) 505.

[8] De Groot, S. R. and MAZUR, P., Non-equilibrium thermodynamics (North-Holland, Amsterdam) 1969, ch. III.

[9] Hall, H. E., J. Phys. C 9 (1976) L-443.

[10] Graham, R., Phys. Rev. Lett. 33 (1974) 1437.

[11] Liv, M., Phys. Rev. B 13 (1976) 4174. 